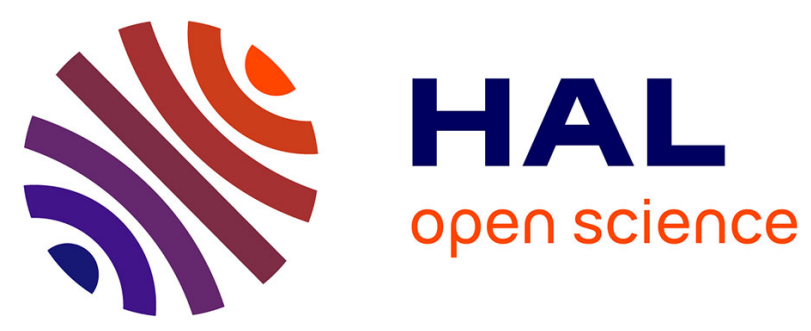

\title{
How to Make nD Functions Digitally Well-Composed in a Self-dual Way
}

\author{
Nicolas Boutry, Thierry Géraud, Laurent Najman
}

\section{To cite this version:}

Nicolas Boutry, Thierry Géraud, Laurent Najman. How to Make nD Functions Digitally WellComposed in a Self-dual Way. Mathematical Morphology and Its Applications to Signal and Image Processing, Benediktsson, J.A.; Chanussot, J.; Najman, L.; Talbot, H., May 2015, Reykjavik, Iceland. pp.561-572, 10.1007/978-3-319-18720-4_47 . hal-01168723

\section{HAL Id: hal-01168723 \\ https://hal.science/hal-01168723}

Submitted on 26 Jun 2015

HAL is a multi-disciplinary open access archive for the deposit and dissemination of scientific research documents, whether they are published or not. The documents may come from teaching and research institutions in France or abroad, or from public or private research centers.
L'archive ouverte pluridisciplinaire HAL, est destinée au dépôt et à la diffusion de documents scientifiques de niveau recherche, publiés ou non, émanant des établissements d'enseignement et de recherche français ou étrangers, des laboratoires publics ou privés. 


\title{
How to Make $n \mathrm{D}$ Functions Digitally Well-Composed in a Self-Dual Way
}

\author{
Nicolas Boutry $^{1,2}$, Thierry Géraud ${ }^{1}$, and Laurent Najman ${ }^{2}$ \\ 1 EPITA Research and Development Laboratory (LRDE) \\ 2 Université Paris-Est, LIGM, Équipe A3SI, ESIEE \\ firstname.lastname@lrde.epita.fr, l.najman@esiee.fr
}

\begin{abstract}
Latecki et al. introduced the notion of $2 \mathrm{D}$ and $3 \mathrm{D}$ wellcomposed images, i.e., a class of images free from the "connectivities paradox" of digital topology. Unfortunately natural and synthetic images are not a priori well-composed. In this paper we extend the notion of "digital well-composedness" to $n \mathrm{D}$ sets, integer-valued functions (graylevel images), and interval-valued maps. We also prove that the digital well-composedness implies the equivalence of connectivities of the level set components in $n \mathrm{D}$. Contrasting with a previous result stating that it is not possible to obtain a discrete $n \mathrm{D}$ self-dual digitally well-composed function with a local interpolation, we then propose and prove a selfdual discrete (non-local) interpolation method whose result is always a digitally well-composed function. This method is based on a sub-part of a quasi-linear algorithm that computes the morphological tree of shapes.
\end{abstract}

Keywords: well-composed functions · equivalence of connectivities · cubical grid · digital topology $\cdot$ interpolation $\cdot$ self-duality.

\section{Introduction}

Connectivities paradox is a well-documented issue in digital topology [7]: a connected component has to have a different connectivity whether it belongs to the background or to the foreground. Well-composed images have been introduced in 2D [9] and 3D [8] to solve that issue, as one of their main properties is the equivalence of connectivities. Intuitively, a major interest of well-composed image is to have functions (values) defined independently from the underlying space structure (graph). Indeed, life is easier if the connectivity of upper and lower level sets are the same. This is especially true when considering self-duality (recall that a transform $\varphi$ is self-dual iff $\varphi(-u)=-\varphi(u)$, where $\varphi$ acts on the space of functions): peaks and valleys are not processed with the same connectivity, and as a consequence the self-duality property is not "perfectly pure". The companion paper [4] discusses at length these questions, which have been largely ignored in the literature on self-duality.

Given that sequences of 3D images become more and more frequent, notably in the medical imaging field and in material sciences, it is important to extend well-composedness in $4 \mathrm{D}$, and more generally in $n \mathrm{D}$. However, extending the notion to higher dimension is not straightforward. The main objective of this paper is twofold. 
- First, we review and study some possible extensions of the well-composedness concept: based on the equivalence of connectivity, based on the continuous framework, based on the combinatorial definition of $n$-surfaces, and, most importantly in this paper, the digital well-composedness, based on some critical configurations. We prove in particular that digital well-composedness implies the equivalence of connectivities.

- Second, we propose a non-local interpolation producing, from a gray-level image defined on the $n \mathrm{D}$ cubical grid, an interpolated digital well-composed $n \mathrm{D}$ image, with $n \geq 2$. Recall that, in the same setting, we have proved [1] that, under some usual constraints, no local self-dual interpolation method can succeed in making $n \mathrm{D}$ digital well-composed functions for $n \geq 3$. Last, let us mention that another approach, based on changing the image values, has been investigated in [2]

The outline of this paper is the following. Section 2 presents several notions of well-composed sets and functions; as a side-effect, it also provides a disambiguation of the multiple definitions of "well-composedness". In Section 3, we propose an extension to $n \mathrm{D}$ of the notion of digital well-composedness, precisely on $n \mathrm{D}$ digitally well-composed sets, functions, and interval-valued maps. Section 4 studies a front propagation algorithm $\mathfrak{F P}$, and states that, when an $n \mathrm{D}$ intervalvalued map $U$ is digitally well-composed, then $\mathfrak{F P}(U)$ is digitally well-composed. In Section 5 we explain how practically we can turn an $n \mathrm{D}$ integer-valued function $u$ into an $n \mathrm{D}$ well-composed function. Last we conclude in Section 6 and gives some perspectives of our work.

Note that, due to limited space, the proofs of theorems and propositions will be given in an extended version of this paper, available on the Internet from http://hal.archives-ouvertes.fr

\section{About Well-Composedness}

\subsection{Well-Composed Sets}

An important result of the paper is the clarification of the terminology and of the various approaches dealing with well-composedness on cubical grids. There exist four different definitions:

- the well-composedness based on the "equivalence of connectivities" (or EoC well-composedness), EWC for short, which is the seminal definition of "wellcomposed 2D sets";

- the digital well-composedness, DWC for short, which relies on the definition of critical configurations (explained later in this paper);

- the continuous well-composedness, CWC for short, which relies on the continuous framework;

- the Alexandrov well-composedness, AWC for short, which relies on the combinatorial definition by Evako et al. [3] of $n$-surface.

As said in the introduction, digital topology is well known to force the practitioners to use a pair of connectivities [7], e.g. in 2D both $c_{4}$ and $c_{8}$. To avoid 


\begin{tabular}{|c|c|c|c|c|c|c|c|}
\hline 2D case: & EWC 9] & $\Longleftrightarrow$ & DWC & $\Longleftrightarrow$ & AWC & $\Longleftrightarrow$ & CWC \\
\hline 3D case: & EWC & $\Longleftarrow$ & DWC & $\Longleftrightarrow$ & AWC & $\Longleftrightarrow$ & CWC 8] \\
\hline$n \mathrm{D}$ case: & EWC & $\Longleftarrow$ & DWC (this paper) & $\Leftarrow ? \Rightarrow$ & AWC [12] & $\Leftarrow ? \Rightarrow$ & CWC [10] \\
\hline
\end{tabular}

Table 1: Different "flavors" of well-composedness: their definition is emphasized (underlined), and the relations between them are depicted. The bottom line is dedicated to their $n \mathrm{D}$ generalizations where $n \geq 2$. Additionally, note that the relation "EWC $\Leftarrow \mathrm{DWC}$ " in $n \mathrm{D}$ comes from this present paper.

this issue, Latecki, Eckhardt and Rosenfeld have introduced the notion of wellcomposed sets and gray-level images in 9 for the 2D case, and shortly afterwards, in [8] for the 3D case. Let us recall these seminal definitions.

Definition 1 (2D well-composed sets, 2D EWC) A $2 D$ digital set $X \in \mathbb{Z}^{2}$ is weakly well-composed if any 8-component of $X$ is a 4-component. $X$ is wellcomposed if both $X$ and its complement $\mathbb{Z}^{2} \backslash X$ are weakly well-composed.

Starting from this definition, denoted by $2 \mathrm{D}$ EWC in Table 1 , the seminal paper [9] shows that it is equivalent to the digital well-composedness, and to the continuous well-composedness. Though, a definition of the 3D well-composedness based on the notion of the "equivalence of connectivities" (3D EWC) does not lead to any interesting topological properties. So, in [8], Latecki introduced the "continuous well-composedness" (CWC) setting:

Definition 2 (3D well-composed sets, 3D CWC) A $3 D$ digital set $X \in \mathbb{Z}^{3}$ is well-composed if the boundary of its $3 D$ continuous analog is a $2 D$ manifold.

As depicted in Table 1 both in $2 \mathrm{D}$ and in $3 \mathrm{D}$, the digital well-composedness, the Alexandrov well-composedness, and the continuous well-composedness are equivalent (this property is used in [6]). A major difference between $2 \mathrm{D}$ and $3 \mathrm{D}$ comes from the fact that, in $3 \mathrm{D}$ and in any greater dimension, we lose the property "EWC $\Rightarrow \mathrm{CWC}$ ". Indeed, the $3 \mathrm{D}$ set $\left(\begin{array}{ll|ll}1 & 0 & 1 & 1 \\ 0 & 1 & 1 & 1\end{array}\right)$ satisfies the "equivalence of connectivities" property - this set is actually both a 26-component and a 6-component-but it is not digitally well-composed.

The continuous well-composedness has been extended in $n \mathrm{D}$ by Latecki (Definition 4 in 10]). Yet, to the best of our knowledge, neither Latecki nor any other author have expanded on this definition. One reason might be that it is difficult to handle from a computational point of view. In contrast, the combinatorial notion of $n$-surface 3 seems highly adapted to model boundaries of subsets of $\mathbb{Z}^{n}$ (or of any subdivision of this space). Following these ideas, a definition of the Alexandrov well-composedness (AWC) has been proposed in [12.

A third approach, based on some forbidden critical configurations, is called the digital well-composedness (DWC), and is the focus of this paper. For practical purposes such as self-duality, the "well-composedness based on the equivalence of connectivities" (EWC) is the property we are looking for. It is a global 
property, while DWC is a local one. In this paper, we define the generalization to $n \mathrm{D}$ of the digital well-composedness (Section 3 ) and we show (Section 3.2) that "DWC $\Rightarrow \mathrm{EWC}$ in $n \mathrm{D}$ ". Hence, DWC is a practical way to check/enforce EWC.

Studying whether or not the continuous well-composedness, the digital wellcomposedness and the Alexandrov well-composedness are equivalent in $n \mathrm{D}$ for $n>3$ is beyond the scope of the paper (in $n \mathrm{D}$, we thus have "AWC $\Leftarrow$ ? $\Rightarrow$ CWC" and "DWC $\Leftarrow ? \Rightarrow$ AWC" in Table 1 .

\subsection{D Well-Composed Functions}

Let us denote by $\mathbb{V}$ a finite ordered set of values; $\mathbb{V}$ can be a finite subset of $\mathbb{Z}$ or of $\mathbb{Z} / 2$.

Given a $2 \mathrm{D}$ image $u: \mathcal{D} \subseteq \mathbb{Z}^{2} \rightarrow \mathbb{V}$ and $\lambda \in \mathbb{V}$, the upper and lower threshold sets of $u$ are respectively defined by $[u \geq \lambda]=\{x \in \mathcal{D} \mid u(x) \geq \lambda\}$ and $[u \leq \lambda]=\{x \in \mathcal{D} \mid u(x) \leq \lambda\}$. The strict threshold sets are obtained when replacing $\geq$ by $>$ and $\leq$ by $<$.

Definition 3 (2D well-composed functions) A function $u$ is well-composed iff all its upper threshold sets are well-composed.

Note that relying on lower threshold sets (instead of upper ones) leads to an equivalent definition. Note that using strict threshold sets (instead of large ones) also leads to an equivalent definition; indeed, in digital topology with a finite set of values, we have: $\exists \epsilon \in \mathbb{R}$ such that $\forall \lambda,[u \geq \lambda]=[u>\lambda-\epsilon]$ and $[u \leq$ $\lambda]=[u<\lambda+\epsilon]$.

A characterization of well-composed 2D images is that, any sub-part of $2 \times 2$ pixels of $u$ valued as $\left(\begin{array}{ll}a & b \\ c & d\end{array}\right)$ shall satisfy $\operatorname{intvl}(a, d) \cap \operatorname{intvl}(b, c) \neq \emptyset$, where $\operatorname{intvl}\left(v_{1}, v_{2}\right)=\llbracket \min \left(v_{1}, v_{2}\right), \max \left(v_{1}, v_{2}\right) \rrbracket$.

\section{Digital Well-Composedness in $n \mathrm{D}$}

This section presents a generalization to $n \mathrm{D}$ of the notions of digitally wellcomposed sets and functions, and an extension of digital well-composedness to interval-valued maps.

\subsection{Notations.}

In the following, we consider sets and functions (typically gray-level images) defined on $(\mathbb{Z} / s)^{n}$, where $s \in\{1,2\}$. When $s=1$ we have the original space $\mathbb{Z}^{n}$, whereas with $s=2$ we have a single subdivision of the original space (every coordinates of $z \in\left(\frac{\mathbb{Z}}{2}\right)^{n}$ are multiple of $\left.\frac{1}{2}\right)$. Practically, the definition domain will always be limited to an hyperrectangle $\mathcal{D} \subset(\mathbb{Z} / s)^{n}$. Let us denote by $\mathbb{B}_{s}$ the canonical basis of $(\mathbb{Z} / s)^{n}$. Given a point $z \in(\mathbb{Z} / s)^{n}$ and a subset $\mathcal{F}=$ $\left\{f_{1}, \ldots, f_{k}\right\} \subseteq \mathbb{B}_{s}$ with $2 \leq k \leq n$, a block associated with $z$ and $\mathcal{F}$ is defined as:

$$
S(z, \mathcal{F})=\left\{z+\sum_{i=1}^{k} \lambda_{i} f_{i} \mid \lambda_{i} \in\left\{0, \frac{1}{s}\right\}, \forall i \in \llbracket 1, k \rrbracket\right\} .
$$




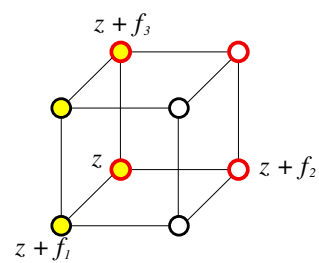

Fig. 1: A block of dimension 3 associated with $z$ contains 8 points of $(\mathbb{Z} / s)^{n}$. Two blocks of dimension 2 associated with $z$ are also depicted, each containing 4 points; their points are respectively filled in yellow and contoured in red. $(z+$ $\left.f_{2}, z+f_{3}\right)$ and $\left(z+f_{1}, z+f_{2}+f_{3}\right)$ are two pairs of antagonist points, respectively for the red $2 \mathrm{D}$ block and for the $3 \mathrm{D}$ block.

Remark that a block $S(z, \mathcal{F}) \subset(\mathbb{Z} / s)^{n}$ actually belongs to a subspace of dimension $k$. In the following, we will thus say a block $S$ of dimension $k$, meaning that we consider a block $S(z, \mathcal{F}) \operatorname{such}$ as $\operatorname{card}(\mathcal{F})=k$ and whatever $z$. Figure 1 depicts some blocks.

Given a block $S \subset(\mathbb{Z} / s)^{n}$ of dimension $k$, and $p, p^{\prime} \in S$, we say that $p$ and $p^{\prime}$ are antagonist in $S$ iff they maximize the distance $L^{1}$ between two points in $S$. Obviously an antagonist to a given point $p \in S$ exists and is unique; it is denoted by $\operatorname{antag}_{S}(p)$. We are now able to generalize the definition of critical configurations to any dimension $n \geq 2$.

\subsection{Digitally Well-Composed $n \mathrm{D}$ Sets.}

A primary critical configuration of dimension $k$ in $(\mathbb{Z} / s)^{n}$, with $2 \leq k \leq n$, is any set $\left\{p, \operatorname{antag}_{S}(p)\right\}$ with $S$ being a block of dimension $k$. A secondary critical configuration of dimension $k$ in $(\mathbb{Z} / s)^{n}$ is any set $S \backslash\left\{p, \operatorname{antag}_{S}(p)\right\}$ with $S$ being a block of dimension $k$.

Definition 4 ( $n \mathbf{D}$ DWC sets) A set $X \subseteq(\mathbb{Z} / s)^{n}$ is digitally well-composed iff, for any $k \in \llbracket 2, n \rrbracket$, and for any block $S$ of dimension $k, X \cap S$ is neither a primary nor a secondary critical configuration.

Notice that the definition of digital well-composedness is self-dual: any set $X \subseteq(\mathbb{Z} / s)^{n}$ is digitally well-composed iff $(\mathbb{Z} / s)^{n} \backslash X$ is digitally well-composed. An image is a priori not digitally well-composed; for instance, the classical graylevel "Lena" image contains 38039 critical configurations.

Let us now present a major result about digital well-composedness for $n \mathrm{D}$ sets.

Theorem 1 (Existence of a $2 n$-path between antagonist points) $A$ set $X \subseteq(\mathbb{Z} / s)^{n}$ is digitally well-composed iff, for any block $S$ and for any couple of points $\left(p, \operatorname{antag}_{S}(p)\right)$ of $X \cap S$, resp. $S \backslash X$, there exists a $2 n$-path between them in $X \cap S$, resp. $S \backslash X$. 
Definition 5 ( $n \mathbf{D}$ EWC sets) $A$ set $X \subseteq(\mathbb{Z} / s)^{n}$ is well-composed based on the equivalence of connectivities (EWC) iff the set of $\left(3^{n}-1\right)$-components of $X$, resp. of $(\mathbb{Z} / s)^{n} \backslash X$, is equal to the set of $2 n$-components of $X$, resp. of $(\mathbb{Z} / s)^{n} \backslash X$.

As corollary, we have the equivalence of all the classical connectivities on a cubical grid for a digitally well-composed set:

Corollary $1\left(n \mathbf{D}\right.$ DWC $\Rightarrow n \mathbf{D}$ EWC) If a set $X \subseteq(\mathbb{Z} / s)^{n}$ is digitally wellcomposed $(D W C)$, then $X$ is well-composed based on the equivalence of connectivities $(E W C)$.

\subsection{Digitally Well-Composed $n \mathrm{D}$ Functions.}

The generalization of digital well-composedness from $n \mathrm{D}$ sets to $n \mathrm{D}$ functions is the same as before.

Definition 6 ( $n \mathbf{D}$ DWC functions) Given $u: \mathcal{D} \subseteq(\mathbb{Z} / s)^{n} \rightarrow \mathbb{V}$ (a gray-level image), $u$ is digitally well-composed iff all its upper threshold sets are digitally well-composed.

Following the characterizations of 2D and 3D well-composed gray-level images given respectively by Latecki in [9] and by the authors in [1], we can now express a characterization of $n \mathrm{D}$ digitally well-composed images. Let us recall that the span operator is defined on $V \subset \mathbb{V}$ by $\operatorname{span}(V)=\llbracket \min (V), \max (V) \rrbracket \in \mathbb{I}_{\mathbb{V}}$, where $\mathbb{I}_{\mathbb{V}}$ denotes the set of intervals on $\mathbb{V}$.

Property 1 (Characterization of $n \mathbf{D}$ DWC functions) Given a gray-level image $u: \mathcal{D} \subseteq(\mathbb{Z} / s)^{n} \rightarrow \mathbb{V}, u$ is digitally well-composed iff, for any block $S$ of dimension $k$ and for any couple of points $\left(p, p^{\prime}\right)$ with $p^{\prime}=\operatorname{antag}_{S}(p)$, we have:

$$
\operatorname{intvl}\left(u(p), u\left(p^{\prime}\right)\right) \cap \operatorname{span}\left\{u\left(p^{\prime \prime}\right) \mid p^{\prime \prime} \in S \backslash\left\{p, p^{\prime}\right\}\right\} \neq \emptyset .
$$

\subsection{Digitally Well-Composed $n \mathrm{D}$ Interval-Valued Maps.}

We call interval-valued map a map defined on $\mathcal{D} \subseteq(\mathbb{Z} / s)^{n} \rightarrow \mathbb{I}_{\mathbb{V}}$. Given an interval-valued map $U$, we define two functions on $\mathcal{D} \rightarrow \mathbb{V}$, its lower bound $\lfloor U\rfloor$

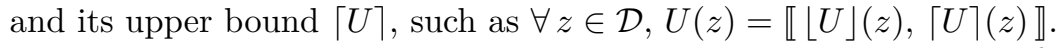

Remark that interval-valued maps are just a particular case of set-valued maps defined on $\mathcal{D} \rightarrow 2^{\mathbb{V}}$ (also denoted by $\mathcal{D} \rightsquigarrow \mathbb{V}$ ). The threshold sets of setvalued maps have been defined in [12]5]; let us recall their definitions, and derive a simple characterization.

$$
\begin{aligned}
& {[U \unrhd \lambda]=\{z \in \mathcal{D} \mid \exists v \in U(z), v \geq \lambda\}, \text { and }[U \triangleleft \lambda]=\mathcal{D} \backslash[U \unrhd \lambda],} \\
& {[U \unlhd \lambda]=\{z \in \mathcal{D} \mid \exists v \in U(z), v \leq \lambda\}, \text { and }[U \triangleright \lambda]=\mathcal{D} \backslash[U \unlhd \lambda], \forall \lambda \in \mathbb{V} .}
\end{aligned}
$$

Definition 7 ( $n$ D DWC interval-valued maps) An interval-valued map $U$ is digitally well-composed iff all its threshold sets are digitally well-composed.

Property 2 (Characterization of $n$ D DWC interval-valued maps) $A n$ $n D$ interval-valued map $U: \mathcal{D} \subseteq(\mathbb{Z} / s)^{n} \rightarrow \mathbb{I}_{\mathbb{V}}$ is digitally well-composed iff both $\lceil U\rceil$ and $\lfloor U\rfloor$ are $n D$ digitally well-composed functions (defined on $\mathcal{D} \rightarrow \mathbb{V}$ ). 


\section{A Study of a Front Propagation Algorithm}

In this section, we study a front propagation algorithm that takes a major role in transforming any $n \mathrm{D}$ function into a digitally well-composed function.

\subsection{Origin of the Front Propagation Algorithm}

The front propagation algorithm studied in this section is related to the algorithm, proposed in [5], which computes in quasi-linear time the morphological tree of shapes of a $n \mathrm{D}$ image. Schematically, the tree of shapes computation algorithm is composed of 4 steps:

$$
u \stackrel{\text { immersion }}{\longrightarrow} U \stackrel{\text { sort }}{\longrightarrow}\left(u^{b}, \mathcal{R}\right) \stackrel{\text { union-find }}{\longrightarrow} \mathcal{T}\left(u^{b}\right) \stackrel{\text { emersion }}{\longrightarrow} \mathcal{T}(u) .
$$

The input is an integer-valued image $u$, defined on the $n \mathrm{D}$ cubical grid. First an immersion step creates an interval-valued map $U$, defined on a larger space $\mathcal{K}$. A front propagation step, based on a hierarchical queue, takes $U$ and produces two outputs: an image $u^{b}$ and an array $\mathcal{R}$ containing the elements of $\mathcal{K}$. In this array, the elements are sorted so that the next step, an union-find-based tree computation, produces $\mathcal{T}\left(u^{b}\right)$ the tree of shapes of $u^{b}$. Actually $\left.u^{b}\right|_{\mathbb{Z}^{n}}=u$ and $\left.\mathcal{T}\left(u^{b}\right)\right|_{\mathbb{Z}^{n}}=\mathcal{T}(u)$. The last step, the emersion, removes from $\mathcal{T}\left(u^{b}\right)$ all the elements of $\mathcal{K} \backslash \mathbb{Z}^{n}$, and also performs a canonicalization of the tree. So $\mathcal{T}(u)$, the tree of shapes of $u$, is obtained [5].

The front propagation step (highlighted in red in the schematic description) acts as a flattening of an interval-valued map $U$ into a function $u^{b}$, because we have $\forall z, u^{b}(z) \in U(z)[5$. In the following, we will denote by $\mathfrak{F P}$ both the front propagation algorithm (the part highlighted in red above) and the mathematical operator $\mathfrak{F P}: U \mapsto u^{b}$.

Last, let us give two important remarks. 1. We are going to reuse the front propagation algorithm $\mathfrak{F P}$, yet in a very different way than it is used in the tree of shapes computation algorithm (see later in Section 5). Indeed, its input $U$ will be different (both the structure and the values of $U$ will be different), and its purpose also will be different (flattening versus sorting). 2. Actually, the front propagation algorithm is just a part of the solution that we present to make $n \mathrm{D}$ functions digitally well-composed.

\subsection{Brief Explanation of the Front Propagation Algorithm}

Let us now explain shortly the $\mathfrak{F P}$ algorithm, which is recalled in Algorithm 1 (see [5] for the original version). This algorithm uses a classical front propagation on the definition domain of $U$. This propagation is based on a hierarchical queue, denoted by $Q$, the current level being denoted by $\ell$. There are two notable differences with the well-known hierarchical-queue-based propagation. First the values of $U$ are interval-valued so we have to decide at which (single-valued) level to enqueue the domain points. The solution is to enqueue a point $h$ at the value of the interval $U(h)$ that is the closest to $\ell$ (see the procedure PRIORITY_PUSH). The image $u^{b}$ actually stores the enqueuing level of the points. Second, when 


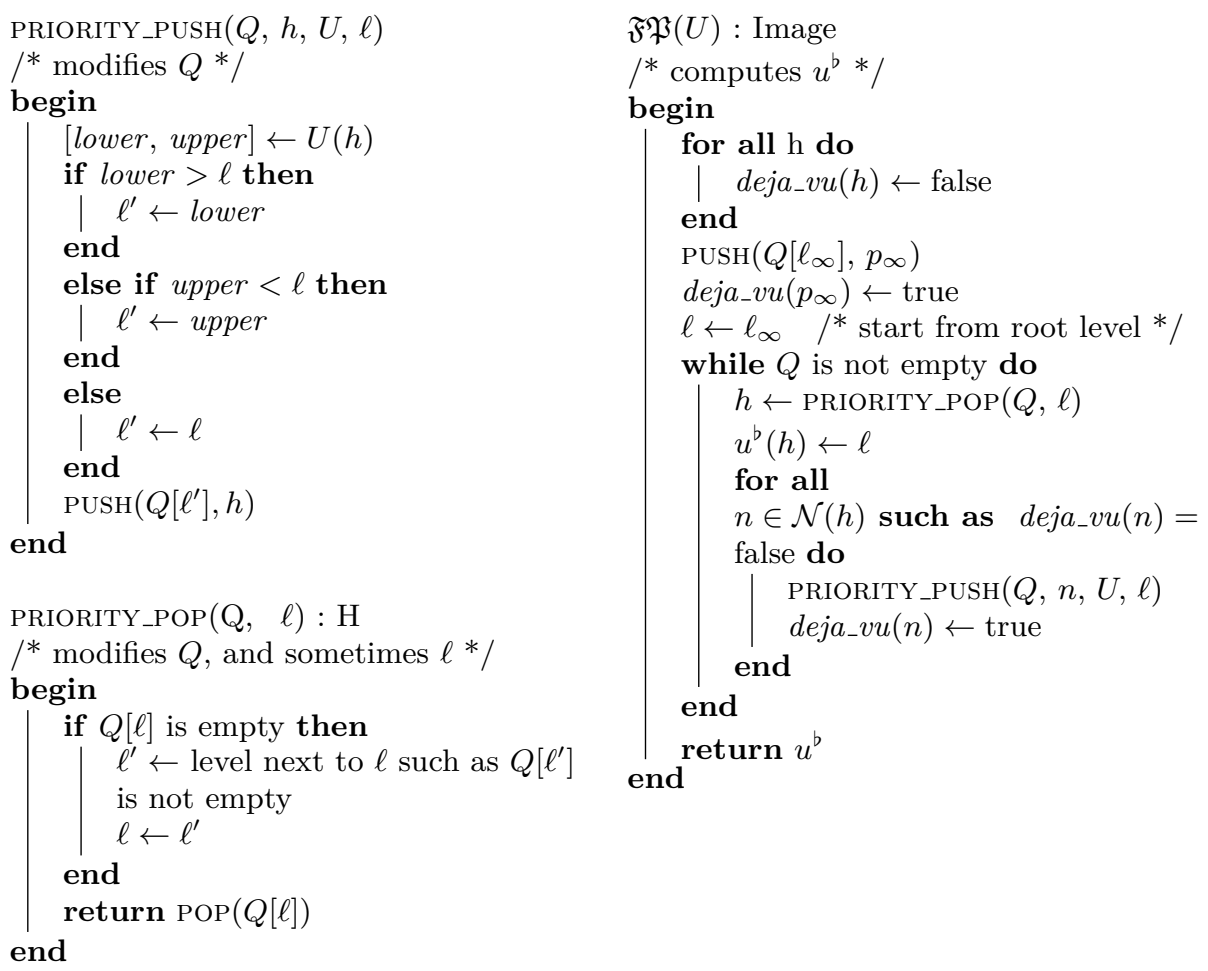

Algorithm 1: Computation of the function $u^{b}$ from an interval-valued map $U$. Left: the routines PRIORITY_PUSH and PRIORITY_POP handle a hierarchical queue. Right: the queue-based front propagation algorithm $\mathfrak{F P}$.

the queue at the current level, $Q[\ell]$, is empty (and when the hierarchical queue $Q$ is not yet empty), we shall decide what is the next current level. We have the choice of taking the next level, either less or greater than $\ell$, such that the queue at that level is not empty (see the procedure PRIORITY_POP). Practically, choosing going up or down the levels does not change the resulting image $u^{b}$. The neighborhood $\mathcal{N}$ used by the propagation corresponds to the $2 n$-connectivity.

Such as in [5], the initialization of the front propagation relies on the definition of a point, $p_{\infty}$ (first point enqueued), and of a value $\ell_{\infty} \in U\left(p_{\infty}\right)$, which is the initial value of the current level $\ell$. Similarly to the case of the tree of shapes computation, $p_{\infty}$ is taken in the outer boundary of the definition domain of $U$. The initial level $\ell_{\infty}$ is set to the median value of the points belonging to the inner boundary of the definition domain of $U$; more precisely, when the interval-valued $U$ is constructed from an integer-valued function $u, \ell_{\infty}$ is computed from the values of the inner boundary of $u$. Using the median operator ensures that $\ell_{\infty}$ is set in a self-dual way: schematically $\ell_{\infty}(-u)=-\ell_{\infty}(u)$. An example is given later in Section 5.1 
Last, let us mention that a run of the $\mathfrak{F P}$ algorithm on a simple intervalvalued map $U$ is illustrated in the extended version of this paper, available on http://hal.archives-ouvertes.fr.

\subsection{Properties of the Front Propagation Algorithm}

The front propagation algorithm has three main properties: it is deterministic, it is self-dual, and its output is an $n \mathrm{D}$ digitally well-composed function if its input is an $n \mathrm{D}$ digitally well-composed interval-valued map.

Proposition 1 (FP is deterministic) Once given $p_{\infty}$ and $\ell_{\infty}$, the front propagation algorithm $\mathfrak{F P}$ (Algorithm 1) is deterministic with respect to its input, the $n D$ interval-valued map $U$.

Proposition 2 (FP is self-dual) For any $n D$ interval-valued map $U$, and whatever $p_{\infty}$ and $\ell_{\infty} \in U\left(p_{\infty}\right)$ now considered as parameters, we have:

$\mathfrak{F P}_{\left(p_{\infty}, \ell_{\infty}\right)}(U)=-\mathfrak{F P}_{\left(p_{\infty},-\ell_{\infty}\right)}(-U)$, so $\mathfrak{F P}$ is self-dual.

Actually, the front propagation algorithm features some continuity properties due to the fact that the front propagation is spatially coherent, and due to the way the hierarchical queue is handled [5]. Consequently, we have for $\mathfrak{F P}$ the following strong result.

Theorem $2(\mathfrak{F P}(U)$ is DWC if $U$ is DWC) If the $n D$ interval-valued map $U$ : $\mathcal{D} \subset\left(\frac{\mathbb{Z}}{2}\right)^{n} \rightarrow \mathbb{I}_{\mathbb{Z}}$ is digitally well-composed, the resulting $n D$ function $\mathfrak{F P}(U)$ is digitally well-composed.

\section{$5 \quad$ Making an $n \mathrm{D}$ Function Digitally Well-Composed}

In this section we present a method to make any $n \mathrm{D}$ integer-valued function $u$ (typically a gray-level image) digitally well-composed. This method is composed of two steps; the first one is an interpolation of $u$ that gives an interval-valued map $U_{\mathrm{Dwc}}$, and the second one is the flattening $\mathfrak{F P}$ that gives the resulting singlevalued function $u_{\mathrm{Dwc}}$ :

$u: \mathcal{D} \subset \mathbb{Z}^{n} \rightarrow \mathbb{Z} \stackrel{\text { interpolation }}{\longmapsto} U_{\text {DWC }}: \mathcal{D}_{2} \subset\left(\frac{\mathbb{Z}}{2}\right)^{n} \rightarrow \mathbb{I}_{\mathbb{Z}} \stackrel{\text { flattening }}{\longmapsto} u_{\text {DWC }}: \mathcal{D}_{2} \rightarrow \frac{\mathbb{Z}}{2}$.

We are looking for an interpolation method that turns any function $u$ into a digitally well-composed map $U_{\mathrm{Dwc}}$, so that eventually $u_{\mathrm{Dwc}}=\mathfrak{F P}\left(U_{\mathrm{Dwc}}\right)$ is a digitally well-composed function (thanks to Theorem 2).

\subsection{Interpolation}

Let us consider a function (gray-level image) $u: \mathcal{D} \subset \mathbb{Z}^{n} \rightarrow \mathbb{V}$. We subdivide the space $\mathbb{Z}^{n}$ into $(\mathbb{Z} / 2)^{n}$, and define a new map on $(\mathbb{Z} / 2)^{n}$. The new definition domain is $\mathcal{D}_{2} \subset(\mathbb{Z} / 2)^{n}$ where $\mathcal{D}_{2}$ is the smallest hyperrectangle such as $\mathcal{D} \subset \mathcal{D}_{2}$. A sensible property of this new map is to be equal to $u$ on $\mathcal{D}$. The values of this new map over $\mathcal{D}_{2} \backslash \mathcal{D}$ are obtained by locally interpolating the values of $u$. With 


\begin{tabular}{|c|c|c|c|c|c|c|c|c|}
\hline$\{8\}$ & $\{8\}$ & $\{8\}$ & $\{8\}$ & $\{8\}$ & $\{8\}$ & $\{8\}$ \\
\hline$\{8\}$ & $\{\mathbf{9}\}$ & $\llbracket 9,11 \rrbracket$ & $\{\mathbf{1 1}\}$ & $\llbracket 11,15 \rrbracket$ & $\{\mathbf{1 5}\}$ & $\{8\}$ \\
\hline$\{8\}$ & $\llbracket 7,9 \rrbracket$ & $\llbracket 1,11 \rrbracket$ & $\llbracket 1,11 \rrbracket$ & $\llbracket 1,15 \rrbracket$ & $\llbracket 13,15 \rrbracket$ & $\{8\}$ \\
\hline$\{8\}$ & $\{\mathbf{7}\}$ & $\llbracket 1,7 \rrbracket$ & $\{\mathbf{1}\}$ & $\llbracket 1,13 \rrbracket$ & $\{\mathbf{1 3}\}$ & $\{8\}$ \\
\hline$\{8\}$ & $\llbracket 3,7 \rrbracket$ & $\llbracket 1,7 \rrbracket$ & $\llbracket 1,5 \rrbracket$ & $\llbracket 3,13 \rrbracket$ & $\llbracket 3,13 \rrbracket$ & $\{8\}$ \\
\hline 9 & 11 & 15 \\
\hline 7 & 1 & 13 \\
\hline 3 & 5 & 3 \\
\hline$\{8\}$ & $\{\mathbf{3}\}$ & $\llbracket 3,5 \rrbracket$ & $\{\mathbf{5}\}$ & $\llbracket 3,5 \rrbracket$ & $\{\mathbf{3}\}$ & $\{8\}$ \\
\hline$\{8\}$ & $\{8\}$ & $\{8\}$ & $\{8\}$ & $\{8\}$ & $\{8\}$ & $\{8\}$ \\
\hline
\end{tabular}

(a) $u$

(b) $\mathcal{I}_{\text {span }}(u)$

Fig. 2: (a): A simple 2D integer-valued function. (b): Its span-based interpolation (the central part of $5 \times 5$ points of $(\mathbb{Z} / 2)^{n}$ with values in $\mathbb{I}_{\mathbb{Z}}$ ); the external border (in gray) is required when passing $\mathcal{I}_{\text {span }}(u)$ to the $\mathfrak{F P}$ algorithm (see text).

$B=\left\{-\frac{1}{2}, 0, \frac{1}{2}\right\}^{n}, B_{z}$ the translation of $B$ by $z$, and "op" an operator on subsets of $\mathbb{V}$, we can define the interpolating map:

$$
\forall z \in \mathcal{D}_{2}, \quad\left(\mathcal{I}_{\mathrm{op}}(u)\right)(z)= \begin{cases}\operatorname{op}\{u(z)\} & \text { if } z \in \mathcal{D}, \\ \operatorname{op}\left\{u\left(z^{\prime}\right), z^{\prime} \in B_{z} \cap \mathcal{D}\right\} & \text { otherwise. }\end{cases}
$$

The following proposition, which could also be derived from [11], follows easily.

Proposition $3\left(\mathcal{I}_{\min }\right.$ and $\mathcal{I}_{\max }$ give dual DWC functions) For any $u: \mathcal{D} \subset$ $\mathbb{Z}^{n} \rightarrow \mathbb{Z}$, the $n D$ integer-valued functions $\mathcal{I}_{\min }(u)$ and $\mathcal{I}_{\max }(u)$ are digitally well-composed, and the interpolation operators $\mathcal{I}_{\min }$ and $\mathcal{I}_{\max }$ are dual (they verify $\forall u, \mathcal{I}_{\min }(u)=-\mathcal{I}_{\max }(-u)$ ).

Since we have $\forall V \subset \mathbb{V}, \operatorname{span}(V)=\llbracket \min (V), \max (V) \rrbracket$, whatever an $n \mathrm{D}$ function $u$, the interval-valued map $\mathcal{I}_{\text {span }}(u)$ is such as $\left\lfloor\mathcal{I}_{\text {span }}(u)\right\rfloor=\mathcal{I}_{\text {min }}(u)$ and $\left\lceil\mathcal{I}_{\text {span }}(u)\right\rceil=\mathcal{I}_{\max }(u)$. Since these two functions are digitally well-composed, the interval-valued map $\mathcal{I}_{\text {span }}(u)$ is digitally well-composed (thanks to Property 2]. So we have:

Proposition $4\left(\mathcal{I}_{\text {span }}\right.$ is self-dual and gives DWC maps) For any $u: \mathcal{D} \subset$ $\mathbb{Z}^{n} \rightarrow \mathbb{Z}$, the $n D$ interval-valued function $\mathcal{I}_{\text {span }}(u): \mathcal{D}_{2} \subset(\mathbb{Z} / 2)^{n} \rightarrow \mathbb{I}_{\mathbb{Z}}$ is digitally well-composed, and the interpolation operator $\mathcal{I}_{\text {span }}$ is self-dual (it verifies $\left.\forall u, \mathcal{I}_{\text {span }}(u)=-\mathcal{I}_{\text {span }}(-u)\right)$.

An example of the span-based interpolation is depicted in Figure 2, The outer/external boundary of the definition domain $\mathcal{D}_{2}$ of $\mathcal{I}_{\text {span }}(u)$ is displayed in gray. This boundary is filled with a single value $\ell_{\infty}(u)$, which is actually the median value of the set of values of the inner/internal boundary of the definition domain of $u$ (see Section 4.2). We have: $\ell_{\infty}(u)=\operatorname{med}\{3,3,5,7,9,11,13,15\}=$ 8. When we take $U_{\text {DwC }}=\mathcal{I}_{\text {span }}(u)$ as input to the $\mathfrak{F P}$ algorithm, $p_{\infty}$ can be any point of the outer boundary. This way, which is similar to [5], we ensure that the propagation starts with the external boundary of $U_{\mathrm{DwC}}$, and that all the points of the internal boundary are enqueued. Having $\ell_{\infty}(-u)=-\ell_{\infty}(u)$ guarantees that " $\mathcal{I}_{\text {span }}$ with an outer boundary added" remains self-dual w.r.t. $u$. 

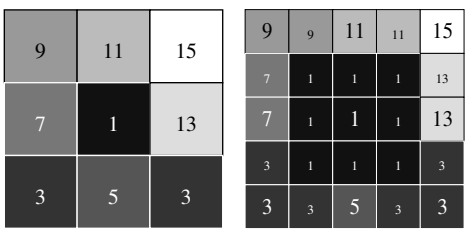

(a) $u$

(b) $\mathcal{I}_{\min }(u)$

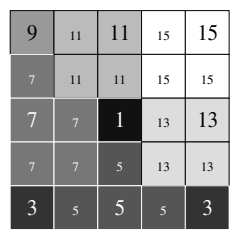

(c) $\mathcal{I}_{\max }(u)$

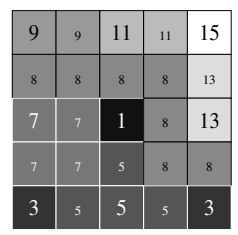

(d) $u_{\text {DwC }}$

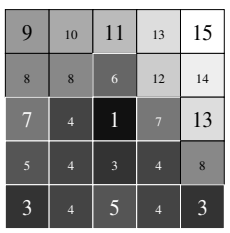

(e) $\mathcal{I}_{\text {med }}(u)$

Fig. 3: Given an integer-valued function $u$, depicted in (a), we have the dual interpolations depicted in (b) and (c), and the self-dual digitally well-composed interpolation $u_{\text {Dwc }}=\left(\mathfrak{F P} \circ \mathcal{I}_{\text {span }}\right)(u)$ depicted in $(\mathrm{d})$. Remark that $\mathcal{I}_{\text {span }}(u)$ is depicted in Figure 2b The rightmost sub-figure (e) depicts a local self-dual interpolation based on the median operator, $\mathcal{I}_{\text {med }}(u)$; this interpolation is digitally well-composed in $2 \mathrm{D}$ but not in $n \mathrm{D}$ with $n>2$ [1]. That contrasts with $\mathfrak{F P} \circ \mathcal{I}_{\text {span }}$, which is a non-local interpolation being digitally well-composed in any dimension.

\subsection{Considering $\mathfrak{F P} \circ \mathcal{I}_{\text {span }}$ as a Solution}

Now, let us consider $u_{\text {Dwc }}=\left(\mathfrak{F P} \circ \mathcal{I}_{\text {span }}\right)(u)$.

Proposition $5\left(\mathfrak{F P} \circ \mathcal{I}_{\text {span }}\right.$ is an $n \mathbf{D}$ self-dual DWC interpolation) Given any $n D$ integer-valued function (gray-level image) $u: \mathcal{D} \subset \mathbb{Z}^{n} \longrightarrow \mathbb{Z}$, the $n D$ function $\left(\mathfrak{F P} \circ \mathcal{I}_{\text {span }}\right)(u): \mathcal{D}_{2} \subset(\mathbb{Z} / 2)^{n} \longrightarrow \mathbb{Z} / 2$ is a self-dual interpolation of $u$ which is digitally well-composed.

$u_{\text {Dwc }}$ is digitally well-composed. We know that $\mathcal{I}_{\text {span }}(u)$ is a digitally wellcomposed map (see Proposition 4), and that $\mathfrak{F P}$ transforms such a map into a digitally well-composed function (see Theorem 2 . Thus $u_{\text {Dwc }}$ is a digitally well-composed function.

$u_{\text {Dwc }}$ is an interpolation of $u$. Since the interpolation $\mathcal{I}_{\text {span }}(u)$ satisfies $\forall z \in \mathcal{D}, \mathcal{I}_{\text {span }}(z)=\{u(z)\}$, and since $\mathfrak{F P}: U \rightarrow u^{b}$ is such that $\forall z \in \mathcal{D}_{2}, u^{b}(z) \in$ $U(z)$, we can deduce that $\left.u_{\mathrm{Dwc}}\right|_{\mathcal{D}}=u$. In addition, the values of $u_{\mathrm{Dwc}}$ in $\mathcal{D}_{2} \backslash \mathcal{D}$ are set "in-between" the ones of $u$ because they belong to their span. Thus $u_{\text {Dwc }}$ is effectively an interpolation of $u$.

$-u_{\text {Dwc }}$ is obtained from $-u$. Both $\mathcal{I}_{\text {span }}$ and $\mathfrak{F P}$ are self-dual (see respectively Propositions 4 and 2 , so we have $\left(\mathfrak{F P} \circ \mathcal{I}_{\text {span }}\right)(-u)=-\left(\mathfrak{F P} \circ \mathcal{I}_{\text {span }}\right)(u)$. The transform $\mathfrak{F P} \circ \mathcal{I}_{\text {span }}$ is therefore self-dual.

As a conclusion, $\mathfrak{F P} \circ \mathcal{I}_{\text {span }}$ is an interpolation method that turns any $n \mathrm{D}$ integer-valued function into a DWC function in a self-dual way. Remark that it is a non-local interpolation method because the interpolated values are set by the propagation of a front in $\mathfrak{F P}$. An illustration is given by Figure 3

\section{Conclusion}

In this paper, we studied several possible extension of the well-composedness concept. In particular, in the framework of digital topology, we prove that dig- 
ital well-composedness implies the equivalence of connectivities. Based on this study, we have shown that a part of the quasi-linear tree-of-shapes computation algorithm produces an interpolated well-composed image. We think that this result is remarkable, contrasting with the fact that it is not possible to obtain a well-composed image in $n \mathrm{D}$ with a local self-dual interpolation [1]. Future work will build on this framework. In particular, the relationships between DWC, AWC, and CWC deserve an in-depth study.

\section{Acknowledgments}

This work received funding from the Agence Nationale de la Recherche, contract ANR-2010-BLAN-0205-03 and through "Programme d'Investissements d'Avenir"

(LabEx BEZOUT $n^{\circ}$ ANR-10-LABX-58). We would also like to warmly thank Michel Couprie for enlightening discussions and the anonymous reviewers for their very insightful comments which have helped us to improve the paper.

\section{References}

1. Boutry, N., Géraud, T., Najman, L.: On making $n \mathrm{D}$ images well-composed by a self-dual local interpolation. In: DGCI. LNCS, vol. 8668, pp. 320-331 (2014)

2. Boutry, N., Géraud, T., Najman, L.: How to make $n \mathrm{D}$ images wellcomposed without interpolation (March 2015), http://hal.archives-ouvertes. fr/hal-01134166

3. Evako, A.V., Kopperman, R., Mukhin, Y.V.: Dimensional properties of graphs and digital spaces. Journal of Mathematical Imaging and Vision 6(2-3), 109-119 (1996)

4. Géraud, T., Carlinet, E., Crozet, S.: Self-duality and discrete topology: Links between the morphological tree of shapes and well-composed gray-level images. In: Proc. of ISMM. LNCS, vol. 9082 (2015), this volume.

5. Géraud, T., Carlinet, E., Crozet, S., Najman, L.: A quasi-linear algorithm to compute the tree of shapes of $n$-D images. In: Proc. of ISMM. LNCS, vol. 7883, pp. 98-110. Springer (2013)

6. Gonzalez-Diaz, R., Jimenez, M.J., Medrano, B.: 3D well-composed polyhedral complexes. Discrete Applied Mathematics 183, 59-77 (March 2015)

7. Kong, T.Y., Rosenfeld, A.: Digital topology: Introduction and survey. Computer Vision, Graphics, and Image Processing 48(3), 357-393 (December 1989)

8. Latecki, L.: 3D well-composed pictures. Graphical Models and Image Processing 59(3), 164-172 (May 1997)

9. Latecki, L., Eckhardt, U., Rosenfeld, A.: Well-composed sets. Computer Vision and Image Understanding 61(1), 70-83 (January 1995)

10. Latecki, L.J.: Advances in Imaging and Electron Physics, vol. 112, chap. WellComposed Sets, pp. 95-163. Academic Press (2000)

11. Mazo, L., Passat, N., Couprie, M., Ronse, C.: Digital imaging: A unified topological framework. Journal of Mathematical Imaging and Vision 44(1), 19-37 (2012)

12. Najman, L., Géraud, T.: Discrete set-valued continuity and interpolation. In: Proc. of ISMM. LNCS, vol. 7883, pp. 37-48 (2013) 